\title{
11. Jahres-Symposium der Berliner Stiftung für Dermatologie und Wissenschaftliche Sitzung anlässlich des 75. Geburtstages von Prof. Dr. Dr. h. c. mult. Constantin E. Orfanos
}

\author{
11th Anniversary Meeting of the Berlin Foundation of Dermatology and Scientific Session \\ Dedicated to 75th Birthday of Prof. Dr. Dr. h.c. mult. Constantin E. Orfanos
}

Autor

Institut

\section{H. Gollnick}

Universitätsklinik für Dermatologie und Venerologie, Medizinische Fakultät der Otto-von-Guericke-Universität, Magdeburg

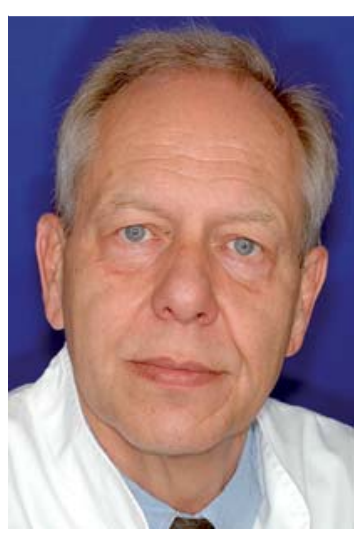

Prof. Dr. med. Harald Gollnick

\section{Bibliografie}

DOI http://dx.doi.org/ 10.1055/s-0030-1257176 Akt Dermatol 2012; 38: 57-58 @ Georg Thieme Verlag KG Stuttgart · New York ISSN 0340-2541

Korrespondenzadresse

Prof. Dr. med.

Harald Gollnick

Universitätsklinik für

Dermatologie und Venerologie Medizinische Fakultät der Otto-von-Guericke-Universität Leipziger Straße 44 39120 Magdeburg harald.gollnick@med.ovgu.de
Beeindruckend - akademisch - festlich - freundschaftlich: Mit diesen Attributen kann man die gemeinsame Veranstaltung der Berliner Stiftung für Dermatologie und der von Prof. Constantin Orfanos und seinen Schülern/innen anlässlich seines 75. Geburtstages ausgerichteten Sitzung am 1. und 2. Juli 2011 in Berlin mit Recht bezeichnen.

Beeindruckend die Verbindung von konzertanter Musik, verbunden mit zwei außerordentlichen Vorträgen zur Haut in der Kunst, und von hohem akademischem Niveau die Festvorträge bei der Berliner Stiftung und die Vorträge anlässlich der wissenschaftlichen Festsitzung. Festlich der Rahmen in der Gemäldegalerie der Staatlichen Museen zu Berlin und die freundschaftliche Atmosphäre und das Miteinander am Sitzungstag. Innerhalb der deutschen Stiftungslandschaft und hier im Besonderen in der Sparte Medizin ist die Berliner Stiftung eine noch junge, aber die älteste in der Dermatologie. Sie feierte nun ihre 11. Jahressitzung. Stiftungen und die sie tragenden Kuratoren, die Stifter und die Zustifter sowie die akademische Welt und Freunde solcher Einrichtungen werden zunehmend treibende Kräfte im Wissenschaftsgeschehen der universitären Gemeinschaft, sind Orte der Versammlung und des Gedankenaustausches, der Ideengebung und des besonderen akademischen Niveaus. Die Berliner Stiftung hat sich in den 11 Jahren ihres Bestehens bewährt und ausgezeichnet (siehe 10-Jahres-Broschüre, 1999-2009). Der Termin des Stiftungsfestes 2011 war koinzident mit dem 75. Geburtstag der Ideengeber und Gründer - seinerzeit zusammen mit Prof. Günter Stüttgen - gewählt worden.

Am Freitag, den 1.7.2011, fanden sich Freunde und Gäste der Stiftung und des Geburtstagskindes zu einem Benefizkonzert als gemeinsamer Veranstaltung der Berliner Stiftung für Dermatologie und der Gemäldegalerie der Staatlichen Museen zu Berlin zusammen. In einem künstlerisch hoch anspruchsvollen Konzert des Orchesters der Kammerakademie Potsdam mit Stücken von A. Vivaldi, J.S. Bach, G.P. Telemann und G.F. Händel war ein musikalischer Rahmen gegeben, der alle zu Beifallsstürmen hinriss. In das musikalische Programm hinein waren zwei Vorträge eingewoben, der eine von Prof. Dr. Bernd W. Lindemann, Direktor der Gemäldegalerie, mit dem Thema „Die Haut in der Kunst“ und der andere von Prof. Dr. Constantin E. Orfanos mit dem Titel „Die Haut in der Medizin“.

Am 2. Juli begann das Wissenschaftliche Symposium der Berliner Stiftung mit einem Festvortrag von Prof. Dr. Dr. h.c. Harald Stein (Berlin) mit dem Titel „Kutane Lymphome. Ihre Evolution und Bedeutung“. Harald Stein, Kuratoriumsmitglied der BSD, bereitete mit seinem Vortrag den Boden für den des Preisträgers Priv.-Doz. Dr. Chalid Assaf (Krefeld). C. Assaf war Doktorand bei H. Stein und erhielt seine Ausbildung bei C.E. Orfanos und später bei W. Sterry. Nun ist er junger Chef der Hautklinik in Krefeld. Sein Thema „Bedeutung von Transkriptionsfaktoren in der Pathogenese maligner Lymphome“ spiegelte seine Jahre dauernde Forschung auf diesem Gebiet wider, die exzellenten Publikationen, und war der Anlass, ihn zum Preisträger des Jahres 2011 der BSD zu wählen. Der Preis war dieses Jahr mit $10000 €$ ausgelobt worden. Anschließend erfolgte die Vergabe der traditionellen BSD-Stipendien an die Herren R. Assefa (Äthiopien) und A. Moubasher (Ägypten).

Der Sitzung der Berliner Stiftung für Dermatologie, die nun zum ersten Mal in den Kongressräumen des „Seminaris Campus Hotels“ am Ort der Freien Universität Berlin in Dahlem stattfand, folgte dann die Wissenschaftliche Sitzung anlässlich des 75. Geburtstages von C.E. Orfanos. Er wurde einleitend durch H. Gollnick und durch einen Festvortrag des Präsidenten der DDG, Prof. Dr. Rudolf Stadler, mit dem Titel „Das Leben eines Wissenschaftlers von der Morphe zur Funktiona- 
lität“ geehrt. Die Schülerschaft der 1., 2. und 3. Generation war zahlreich aus Deutschland und dem Ausland seinem Ruf zu Ehren gefolgt. C.E. Orfanos wurde als Forscher und Lehrer, als Klinker und Arzt, als Erzieher und Ratgeber und als eine Persönlichkeit von uns erlebt, geprägt durch verstandenen und gelebten Humanismus, gewandt in der Kultur der Sprachen, geprägt von tiefem Wissen und Verständnis der Kunst in der Antike und der Moderne, der klassischen Musik und der Oper. Wer C.E. Orfanos über viele Jahre oder Jahrzehnte kennt, weiß, wie gut er in der Dialektik geschult und im geschliffenen Wort gewandt ist. Man kennt ihn als einen Mann, der kein Gefecht scheut, dessen Blicke Blitze schleudern, aber auch große Wärme ausstrahlen können. Er hat sich während seines akademischen Lebens stets durch seinen starken Willen ausgezeichnet, kontinuierlich vorwärts zu streben und die gesetzten Ziele einzuhalten, Visionen zu folgen und Neues am Horizont rasch zu entdecken, anzunehmen, zu entwickeln und sich zu eigen zu machen. Wir erleben seine große Gastfreundschaft, die ein bedeutendes Gut für ihn darstellt. Oft und auch heute schlagen zwei Herzen in seiner Brust, das eine, das deutsch geworden ist, das preußischen Rhythmus in sich trägt, und das andere, das ihn stets an Kreta und die griechische Heimat erinnert, die Ägäis und das gleißende Licht, welches sich vom weißen Marmor der antiken Stätte reflektiert.

Seine Schüler haben innerhalb der Sitzung zwei seiner Schwerpunkte aus der Berliner Hautklinik an der FU ausgewählt, zum einen die dermatologische Onkologie und zum anderen die dermatologische Pharmakologie. In einer dritten Sitzung wurde von seinen im Ausland lebenden Schülern das internationale Engagement in der Dermatologie gewürdigt.

Unter Leitung von Prof. Sergij Goerdt (Mannheim) wurden die „Keratinozytären Tumoren: Herausforderungen und Chancen“ von Prof. Martin Leverkus (Mannheim/vormals Magdeburg und Würzburg) besprochen, „Apoptoseresistenz beim kutanen TZell-Lymphom“ von PD Dr. C.-D. Klemke (Mannheim) und dann
„Tumor und Gerinnung: Fluch oder Segen“ von Prof. S. W. Schneider (Mannheim).

In der Sitzung Dermatologische Pharmakologie unter der Leitung von Prof. C. C. Zouboulis (Dessau) sprach Prof. M. Schmuth (Innsbruck) über „Nukleäre Rezeptoren und moderne Dermatotherapie“, in Vertretung von Priv.-Doz. Dr. A. Thielitz (Magdeburg) H. Gollnick über „Ektopeptidasen: Von der Forschung in die Klinik“ und Prof. M. Schön (Göttingen) schloss diese Sitzung mit dem Thema „Psoriasisforschung und Zukunft der Psoriasistherapie“ ab.

Im Internationalen Symposium unter der Leitung des Tagungsleiters Prof. Dr. Dr. C. Geilen (Berlin) berichtete B. Kurbanov (Berlin) aus seiner Promotion zu „Apoptose und Melanom: Neue therapeutische Zielstrukturen“, Prof. K. Krasagakis (Heraklion/ Griechenland) über „Neue pathogenetische Aspekte beim Merkelzellkarzinom“ und Prof. J. Masenga (Moshi/Tansania) über das „Kaposi Sarcoma in Africa“.

Mit den wohlgesetzten, von langer akademischer Lebenszeit geprägten und von philosophischen Gedanken getragenen Worten „Ein Jegliches hat seine Zeit..." von Prof. Dr. E. C. Jung (Heidelberg), dem ehemaligen Direktor der Universitätshautklinik Mannheim und ehemaligen Herausgeber dieser Zeitschrift, fand die Sitzung einen würdigen Abschluss.

Akademische Feiern in neuer Tradition ohne Fesseln historischer Etikette, getragen von exzellenten wissenschaftlichen Vorträgen, aufgenommen und erlebt durch Schüler schon dreier Generationen des Geburtstagskindes C.E. Orfanos sind in unserem Fach neue Orte der Begegnung, fern von der Hektik und dem Lärm großer Kongresse, losgelöst von der Arena des Wettkampfes sie sind ein neuer Platz, in dem wir uns im Gespräch wiederfinden, gemeinsame Ziele sehen und miteinander aufnehmen, uns über die Grenzen des Faches hinaus austauschen und die Freundschaft in der Akademia pflegen können. 